\title{
A Note to the Reader
}

Almost all good writing begins with terrible first efforts. You need to start somewhere.

-Anne Lamott, Bird by Bird: Some Instructions on Writing and Life

When it comes to writing science proposals, figuring out where to start is always a challenge. We wrote this book to be read two ways: either in its entirety before beginning to write a proposal or chapter by chapter-but not necessarily in sequence-as you develop specific sections of your proposal.

The schematic shown here illustrates the steps of proposal development and writing. You begin with an idea, but sometimes a solicitation (or request for proposals) from a particular agency can provide motivation. There are many steps between the formation of this initial idea and successfully obtaining funding to conduct your research. The process is an iterative one that allows your co-authors, 


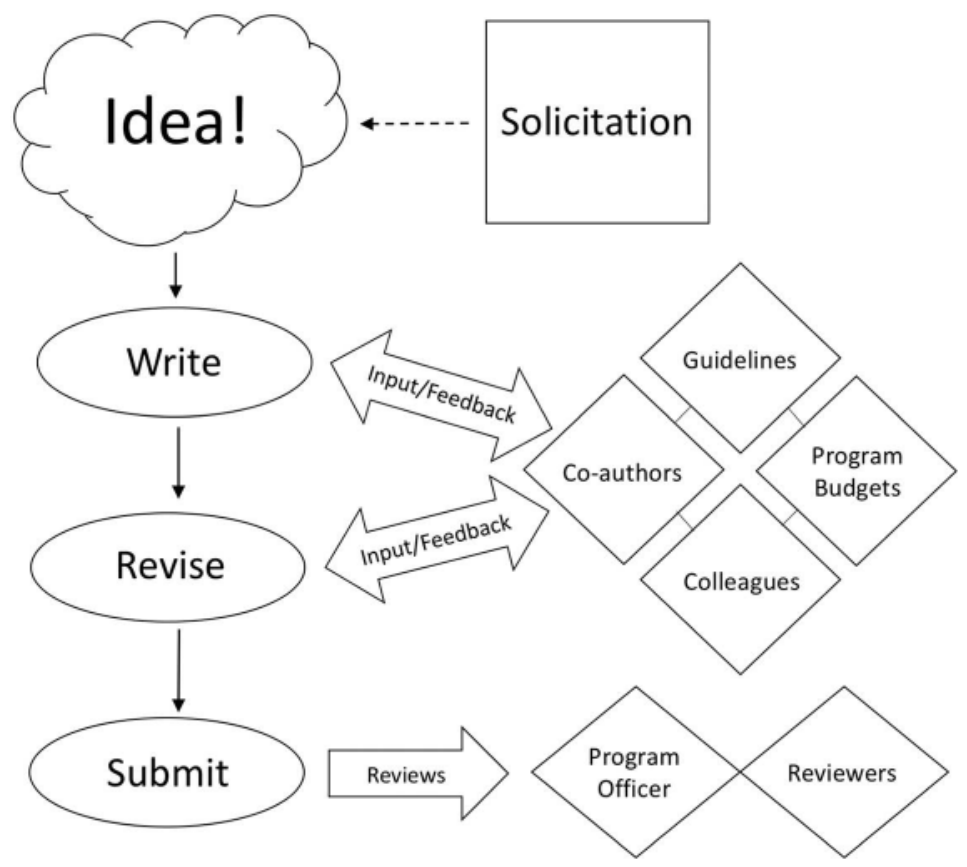

colleagues, reviewers, and funding agency personnel to provide input that you will use to refine and even revise the proposal. Agency guidelines and budgetary restrictions will also influence the development of your proposal. Once you have reconciled all input and feedback, you submit the final document.

The process may seem simple, but over years of developing our own research proposals and teaching our course, Design and Development of Scientific Proposals, we've identified several common areas in which researchers often struggle 
when it comes to writing successful research proposals. Using this book to hone your skills will improve your ability to:

- Identify and describe the conceptual framework of the research question

- Review the relevant literature, both theoretical and empirical, on the system to be studied and any systems related to it

- Describe a general research question in the context of the conceptual framework and the theoretical and empirical work that precedes the proposed work

- Formulate a concise and incisive set of hypotheses or specific aims to address the overarching question

- Design studies to test each hypothesis or aim

- Develop methods and techniques to test, analyze, and synthesize results

- Evaluate potential alternative outcomes that may be obtained from each part of the study and consider where each of these alternatives may lead

- Combine these items-framework, literature review, hypotheses, etc.-into a coherent, precise, concise, and exciting proposal

- Submit the proposal to the appropriate agency or evaluation committee 
- Interpret and respond constructively to reviews of the proposal

The chapters in this book address these common areas of difficulty and provide detailed guidelines for writing grant applications. We present the material in much the same order we have used in teaching our course and in designing our own research proposals. Although we focus on research proposals for the natural sciences, the process and logic presented here apply to a variety of disciplines, including those in the social sciences.

In Chapters 1 and 2 we discuss how to start writing a proposal, how to identify funding opportunities, and how to determine the roles of collaborating authors. In Chapter 3 we outline the basic elements and organization of a proposal. In Chapter 4 we consider the conceptual framework and how and where in the proposal to articulate succinctly the study's significance. In Chapters 5 through 13 we address the requirements and construction of the specific elements found in most standard proposals, such as title, summary, background, methods, and budget. We present the mechanics of submitting and tracking a proposal and revising and resubmitting it in Chapters 14 and 15. In Chapter 16 we discuss submitting proposals to private foundations. In Chapter 17 we look at the particular needs of multidisciplinary, multiinvestigator proposals. In Chapter 18 we conclude the book with some thoughts about ethics and scientific research. 
Each chapter contains a list of key points and suggested homework assignments or practice activities to reinforce the material covered. We kept the lists of key points short to help you retain the major ideas presented in each chapter. We designed the homework assignments so you can use them either in sequence while writing your proposal or chapter by chapter when you need practice with certain parts of the process. You may also find helpful the lists of additional resources and useful links located at the end of the book.

If you submit a proposal after using this book or if you use this book in a course, please let us know how you fare. We can be reached at wssp3e@gmail.com. We look forward to hearing from you and wish you great success with your science proposals. 